\title{
Association of Perioperative Cell-free DNA (cfDNA) Concentrations with Risk of Recurrence in Patients with Breast Cancer
}

Fara Hassan ( $\nabla$ farahassankhawaja@rcsi.ie)

University College Cork https://orcid.org/0000-0002-4021-2054

Jiang Huai Wang

University College Cork

Carolyn Cullinane

Cork University Hospital

Michael Ita

University College Cork College of Medicine and Health

Mark Corrigan

Cork University Hospital

Donal Peter O'Leary

Cork University Hospital

Henry Paul Redmond

Cork University: University College Cork

\section{Research article}

Keywords: Breast cancer, perioperative period, cell free DNA (cfDNA), cfDNA concentrations, liquid biopsy, preoperative period, post-operative period, Disease free(DF) breast cancer, Disease recurrence (DR) breast cancer, recurrence, recurrence free survival (RFS), surgery, prognosis

Posted Date: November 22nd, 2021

DOI: https://doi.org/10.21203/rs.3.rs-1017958/v1

License: (c) (i) This work is licensed under a Creative Commons Attribution 4.0 International License.

Read Full License 


\section{Abstract}

Background: Circulating cell-free DNA (cfDNA) is a potential biomarker of disease status in cancer patients and provides valuable diagnostic and prognostic information in breast cancer. In this study, we sought to quantify the cfDNA concentrations in the perioperative period and to investigate its prognostic relevance in breast cancer patients.

Methods: Sixty-three $(n=63)$ breast cancer patients undergoing curative surgery were screened for inclusion. Blood samples were collected: pre-operatively (Pre-op), post-operatively (POD) within weeks 1-2, weeks 3-4 and weeks 5-12 following surgery. cfDNA was extracted and quantified using nanodrop spectrophotometer. All patients were followed up for 5 years.

Results: The median age was 52(26-84) years. During the perioperative period, patients with high cfDNA concentrations(cutoff:480ng/ml) had inferior recurrence free survival (RFS) than those with lower cfDNA concentrations (pre-operative period: median RFS: 30(3-60) months versus 60(6-60) months $(p<0.0001)$, post-operative period: median RFS: $24(3-60)$ months versus $60(6-60)$ months $(p<0.0001)$. Multivariate Cox regression analysis showed that post op cfDNA concentration $(p=0.017)$, subtypes $(p=0.011)$ and tumour size $(p=0.006)$ were negative prognostic factor for RFS in the pre-operative period and postoperative period.

Conclusion: Our study demonstrated the prognostic ability of perioperative cfDNA concentrations in breast cancer patients. Further, prospective studies are warranted to validate its clinical utility in breast cancer.

\section{Introduction}

Breast cancer is one of the most common malignancies diagnosed among women globally. Although early detection and improved adjuvant therapies are often successful, the 5-year survival rate of breast cancer patients who develop metastatic disease is only $24 \%$. Early detection of breast cancer i.e., prior to metastatic spread is of crucial importance to patient survival. At present, imaging modalities available for the diagnosis and surveillance of breast cancer include mammography, although it is limited by its sensitivity and false positive rates. Serum markers such as cancer antigen 15-3 (CA15-3) are often employed by oncologists for longitudinal monitoring of disease progression in breast cancer. However, these markers have a long half-life and lack sensitivity as well as specificity, especially in early or localized disease.

Liquid biopsy is a minimally invasive test undertaken using a sample of blood to assess for the presence of cancer cells, nucleic acids or soluble antigens originating from a malignant tumour harboured by the host. The presence of cfDNA in human blood was first reported in 1948 by Mandel and Metais. Leon et al demonstrated in their study that concentrations of cfDNA was elevated in patients with cancer. The most widely reported mechanisms for the release cfDNA into circulation include apoptosis and cell necrosis along with other putative mechanisms such as active secretion and vesicular shedding into the 
bloodstream ${ }^{12}$. Fragments of cfDNA tend to be short, ranging between approximately $130-180$ base pairs in length". The estimated half-life of cfDNA in circulating blood varies from several minutes to 1-2 $\mathrm{h}$.

Quantification of circulating cfDNA has emerged as a possible tool for the early diagnosis and prognosis of various cancers'. Increased levels of circulating cfDNA are frequently associated with an adverse prognosis in cancers. There is some degree of uncertainty regarding the acceptable physiological range of cfDNA, and it is generally accepted that the quantitative and qualitative dynamics of cfDNA are more informative.

The perioperative period has been demonstrated to be crucial in determining the risk of post-operative recurrence in cancer. Our group previously demonstrated that cfDNA levels in colon cancer patients are significantly reduced after surgery, but patients who went on to develop disease recurrence within 2 years may be identified by a rise in their cfDNA levels at day 5 following surgery. To date, the evaluation of perioperative dynamics of cfDNA in breast cancer has been underexplored. Thus, the objective of this study was to quantify cfDNA concentrations in the blood of breast cancer patients in the peri-operative period. Furthermore, we sought to investigate the prognostic relevance of peri-operative cfDNA quantitative dynamics in breast cancer patients.

\section{Materials And Methods}

\section{Patient selection, sampling, and follow-up}

Breast cancer patients undergoing curative surgery at Cork University Hospital, Cork, Ireland were screened for inclusion. Blood samples from these patients were stored in a prospectively maintained biobank at Cork University Hospital. Written informed consent was obtained from all patients who participated in this study. Ethical approval for this project was granted by the Clinical Research Ethics Committee (CREC), University College Cork Teaching Hospitals (ECM 3 (iii) 22/10/19 and ECM 4(m) $11 / 01 / 11)$

To study perioperative cfDNA dynamics in breast cancer, we enrolled breast cancer patients from the prospectively maintained biobank. Sixty-three patients undergoing elective surgery for stage I-III breast cancer at Cork University Hospital were enrolled in the study. Paired blood samples were collected for each patient in the preoperative and post-operative period. The preoperative samples were collected on the day before undergoing surgery. The post-operative samples were collected in three time periods (week 1-2, week 3-4 and week 5-12) after surgery. All patients were followed up for at least 5 years to document recurrence free survival. Relevant patients and tumour characteristics were recorded. All patients underwent standard management as per American Joint Committee on Cancer (AJCC) guidelines. All patients had been followed up following standard breast cancer surveillance strategies. Follow-up of breast cancer patients included clinical examination and yearly mammogram and/or other imaging/laboratory studies were indicated to identify those at risk of recurrence.

\section{Serum extraction from blood samples}


Patient blood samples were collected in 3-ml clot activator blood collection tubes (VACUETTE®). Blood samples were then centrifuged (Centrifuge 5810 R, Eppendorf) at 3,000 rpm for 20 minutes at room temperature. Serum was extracted, stored in labelled aliquots and frozen (Haier Bio-Medical,) at $-80^{\circ} \mathrm{C}$ within 2 hours of blood sample collection.

\section{Extraction and quantification of cfDNA}

The cfDNA was extracted from $1 \mathrm{ml}$ serum samples using the QIAamp circulating nucleic acid kit (Qiagen QIAamp Circulating Nucleic Acid Kit. Cat No. ID: 55114,Venlo, Netherlands) following four principal steps of lysis, binding, washing and elution. Briefly, lysis was performed through the addition of proteinase $\mathrm{K}$ and heated to $60^{\circ} \mathrm{C}$ with added buffer ACL containing carrier RNA to bind nucleic acids and inactivation of DNase and RNase for release of nucleic acids. The lysate was then added to the vacuum column containing a silicone membrane with buffer ACB. The vacuum was applied, and the lysate was pulled through the column by vacuum. Three cycles of washing were then performed using buffer AVE to remove contaminates and allow binding of nucleic acids to the silicone membrane. Buffer AVE was passed through the vacuum column to elute nucleic acids from the silicone membrane. Quantification of nucleic acids was performed using DS-11 Spectrophotometer (DeNovix DS-11 FX+ with the NanoDrop ${ }^{\mathrm{TM}}$ One spectrophotometer, Wilmington, Delaware, United States). Double-stranded DNA (ds-DNA) programme was selected to calculate nucleic acid concentration and purity. At least three samples with concordance results within $10 \%$ were analysed and the mean of the three measurements used for analysis. If a result was $>10 \%$ discordant from the other samples, a fourth sample was performed and the mean of three concordant samples utilised. Purity for nucleic acid measurement was accepted as a $260 / 280$ ratio $\approx 1.8-2.0$ and a $260 / 230$ ratio $\approx 2.2$.

\section{Statistical Analysis}

Patient demographics and tumour characteristics were summarised using counts and percentages for categorical variables and analysed using $\mathrm{CHI}$ square test or Fischer exact test. The significance of association between cfDNA concentrations in disease-free (DF) and disease-recurrence (DR) breast cancer groups in perioperative period was assessed using t test and reported as mean \pm standard deviation (SD). Cut-off value, sensitivity, and specificity of cfDNA concentration were analysed with Receiver operator curve (ROC) using IBM SPSS version26 software. Co proportional analysis was used to assess the effect of variables on survival in breast cancer. Cox Log rank analysis and Kaplan-Meier survival curve was used to analyse recurrence-free survival in breast cancer patients. Differences were judged to be statistically significant when the $p$ value was less than 0.05 . Data was analysed using Graph Pad Prism version 8.0 (San Diego, CA).

\section{Results}

\section{Clinico-pathological characteristics:}


A total of sixty-three breast cancer patients were enrolled in study. Nineteen patients $(19 / 63,30 \%)$ developed recurrence, while 44 patients $(44 / 63,70 \%)$ remained disease free on follow up assessment. Clinical and tumour characteristics of the patients are summarized in the Table 1. The median (interquartile range) follow-up time for all breast cancer patients was 96 months (12-120) and median time to recurrence was 16(3-48) months. Overall, significant difference was observed only in subtype of breast cancer in those that developed disease recurrence (DR) compared to those who remained disease free (DF) on follow up (Table 1).

\section{Perioperative dynamics of cfDNA concentrations in breast cancer}

Regarding dynamics of cfDNA concentrations in perioperative period, we observed higher concentrations of cfDNA in the pre-operative period (mean \pm SD: $439+/-190.4 \mathrm{ng} / \mathrm{ml}$ ) consistent with presence of tumour burden. Post-surgery, there was a drop in cfDNA concentrations after removal of macroscopic tumour burden as evident by cfDNA concentrations in the first 2 weeks following surgery with a subsequent rise again from week 3 post surgery (mean \pm SD: week 1-2, 410.6+/-160.5 ng/ml, week 3-4, 431.1+/-196 $\mathrm{ng} / \mathrm{ml}$, week 5-12, 445.4+/-184.8ng/ml) (Figure1).

Interestingly, on subgroup analysis, significantly higher cfDNA concentrations were observed in those at risk of future recurrence pre-operatively (mean \pm SD: DF: $378.3+/-143.7$ versus DR: $580.3+/-213.3 \mathrm{ng} / \mathrm{ml}$, $p<0.001$ ) as well as post-operatively (DF: $371.2+/-131.2$ versus DR: $591.3+/-171.0 \mathrm{ng} / \mathrm{ml}, \mathrm{p}<0.001$ ) (Figure2a). Moreover, we observed significant difference in cfDNA concentrations among those at risk of future disease recurrence compared to disease free breast cancer within first week following surgery and this trend continued over the subsequent weeks (mean \pm SD: week 1-2 ( $p=0.003)$, week 3-4 $(p=0.02)$, week 5-12 ( $p=0.005)$ (Figure 2b).

\section{Prognostic value of perioperative cfDNA concentrations in breast cancer}

Cox regression hazard model was used to assess the prognostic effect of various variables on breast cancer recurrence. On univariate analysis, Tumour size,T stage and subtypes along with cfDNA concentration in perioperative time period were found to have statistically mpact on survival. However, on multivariate analysis, post op cfDNA concentrations, subtypes and tumour size were found to have significant effect on recurrence free survival (Table 2). The prognostic value of cfDNA concentrations was also assessed with receiver operative curve (ROC) analysis. ROC analysis revealed that using the cut off value of $480 \mathrm{ng} / \mathrm{ml}$ could discriminate those at risk of disease recurrence from disease free breast cancer patients in both the pre-operative period (sensitivity: $74 \%$, specificity: $82 \%$, AUC: $0.78,95 \%$ confidence interval: 0.64-0.91) and post-operative period (sensitivity: 79\%, specificity: $80 \%$, AUC: 0.84, 95\% confidence interval: 0.64-0.91) (Figure 3a and 3b). Kaplan-Meier survival curves were plotted to further analyse the prognostic ability of cfDNA concentrations on recurrence free survival. The median recurrence free survival in patients with high cfDNA concentration was 34.7+/-21.6 months versus $55.9+/-12.8$ months $(\mathrm{p}<0.001)$. Survival analysis indicated that cfDNA concentration (cut off $480 \mathrm{ng} / \mathrm{ml}$ ) 
in the post operative period specifically was an independent negative prognostic factor for recurrence-free survival (RFS) (Pre-operative period (Univariate: hazard ratio:6.8, 95\% Confidence Interval: 2.4-19.2, p<0.0001, Multivariate: hazard ratio:1.55, 95\% Confidence Interval:0.26-9.10, p=0.62) and (Post-operative period (Univariate: hazard ratio:9.005, 95\% confidence interval: 2.97-27.29, $p<0.0001$, Multivariate: Hazard ratio:11.6, 95\% Confidence Interval:1.54-88.3, p=0.017) (Figure 4a and 4b)

\section{Discussion}

The oncological impact of surgical injury during the perioperative period has been demonstrated to be crucial in determining the risk of post-operative recurrence in cancer. Around $30 \%$ of breast cancer patients present with disease recurrence at follow-up assessment. Indeed, the risk of disease recurrence is highest during the first 5 years (10.4\%), with a peak occurring between years 1 and 2 (15.2\%). Early detection of recurrence is crucial to survival in breast cancer. Identification of reliable and cost-effective assays from an easily accessible source, such as blood could have an inherent role in routine testing for recurrence risk and adjuvant treatment requirements in surgically resectable cancer. Circulating nucleic acids in the form of cfDNA released by tumour cells may provide crucial information relevant to several aspects of cancer biology. Quantification of cfDNA is a simple non-invasive blood-based test and appears to have diagnostic and prognostic relevance in breast cancer' '. In this study, we sought to explore whether peri-operative cfDNA quantification holds any prognostic significance as it relates to disease recurrence in breast cancer patients undergoing curative surgery. We observed that high cfDNA concentrations in the peri-operative period and after surgery correlate with shortened recurrence free survival (RFS).

In breast cancer, optimum timings for measurement of cfDNA in perioperative period are unclear. So, we study dynamics of cfDNA concentrations and its association with outcome of recurrence in breast cancer before and after surgery. In the preoperative period, high concentrations of cfDNA were observed consistent with the presence of tumour burden in breast cancer patients with subsequent drop in cfDNA levels following surgery. Agassi et al and by Lin et al reported similar findings in breast cancer and oral cancer respectively. Intriguingly, significant correlation was observed upon subgroup analysis, between high cfDNA concentrations in the pre-operative period and inferior recurrence free survival in our cohort of breast cancer patients. Bastian et al and $\mathrm{He} \mathrm{Y}$ et al' have also reported an association between preoperative cfDNA concentration and increase risk of recurrence in prostate cancer and colorectal cancer. Following cancer surgery, identification of patients at high risk of recurrence is imperative for adjuvant therapy decisions. Overall, we observed a fall in cfDNA levels following surgical removal of tumour burden. Importantly, in first week after surgery, there was increase in cfDNA concentration among those at higher risk of disease recurrence compared to those who were at lower risk of recurrence. These findings were also observed by our group in a cohort of colon cancer patients where cfDNA concentrations from 48 hours to day 5 after surgery predicted those at risk of recurrence at 2 years. Another study by Hu et al highlighted the significant association between higher cfDNA concentrations on post-operative day 30 and increased risk of disease recurrence in lung cancer patients. The possible 
explanation for high cfDNA concentrations in cancer patients could arise from accumulation of DNA derived from the primary tumour and metastatic deposits in blood. This combined with the reduced clearance of cfDNA contribute to cfDNA elevation in the blood of patients with cancer. Therefore, monitoring concentrations of cfDNA following surgery could aid clinicians in assessing risk stratification during follow up

The diagnostic and prognostic value of cfDNA in breast cancer has been demonstrated in the available literature. However, there is limited evidence about its significance in the context of the peri-operative period in breast cancer. Although the number of patients in our study was small, we observed that cfDNA concentrations in the peri-operative period could discriminate among patients at risk of recurrence with reasonable sensitivity and specificity. In addition, there was a positive correlation between high cfDNA concentration and shortened recurrence-free survival in breast cancer. We highlighted the significance of cfDNA concentrations in the peri-operative period in breast cancer in our proof-of-concept study but it needs to be validated in larger cohort of patients.

Our study provides insights into the dynamics of cfDNA specific to the perioperative period in breast cancer in detecting and characterizing localized disease before clinical symptoms or radiological evidence of progression. The future of this work will involve exploration of different aspects of the cfDNA biology in the context of immediate peri-operative period with objectives and strategies to minimize perioperative surgical stress response which might affect long-term outcomes in breast cancer. Recently, researchers also explored other aspects of cfDNA including combined mutation detection in cfDNA with serum protein biomarkers, machine learning model DELFI (DNA evaluation of fragments for early interception) to incorporate genome-wide fragmentation of cfDNA to identify differences in cfDNA fragment lengths between cancer and non-cancer participants and use of targeted methylation analysis of cfDNA to detect and localize multiple cancer types across all stages at high specificity.

There are some limitations to our study. The sample size of 63 patients was small. To evaluate the effect of perioperative cfDNA concentrations on breast cancer in our study, we only enrolled cohort of breast cancer patients with known 5-year recurrence outcomes (DF and DR subgroups) and availability of paired perioperative blood samples. Furthermore, there is heterogeneity in patients' subgroups and third of patients had neoadjuvant therapy. However, there is now a platform to investigate this research even further in a larger prospective study group. The role of cfDNA for prediction of recurrence in cancer is one of the most challenging endeavours, so future research should focus on defining the time points in the perioperative period as well as standardizing cfDNA assessment techniques to approve its clinical utility as biomarker in breast cancer.

\section{Conclusion}

Our study has illustrated the feasibility of quantitative cfDNA concentrations as a non-invasive and reproducible technique for predicting prognosis in breast cancer patients in the peri-operative period. Thus, peri-operative cfDNA concentrations could be used as a surrogate marker to improve risk 
stratification of breast cancer patients but the clinical validity of liquid biopsy needs to be addressed in large scale randomised control trials to confirm its everyday utility in breast cancer patients.

\section{Declarations}

\section{Consent for publication:}

Not applicable

\section{Acknowledgements:}

We wish to thank all cancer patients who participated in the study. We also wish to thanks to oncology nurses in Cork University Hospital with the collection of samples and Prof. Young for access to biobank for breast cancer patient blood samples.

\section{Author Contributions:}

Ms. Fara has full access to all the data in the study and takes responsibility for the integrity of the data and the accuracy of the data analysis.

Concept and design: All authors.

Acquisition, analysis, or interpretation of data: Hassan, Ita

Drafting of the manuscript: Hassan.F, J.H Wang, Ita, O'Leary, Cullinane. C

Critical revision of the manuscript for important intellectual content: All authors.

Statistical analysis: Hassan, Ita

Supervision: Wang, Corrigan, Redmond

\section{Ethics approval and consent to participate and publication}

Written informed consent was obtained from all patients who participated in this study. Ethical approval for this project was granted by the Clinical Research Ethics Committee (CREC), University College Cork Teaching Hospitals (ECM 3 (iii) 22/10/19 and ECM 4(m) 11/01/11

\section{Funding/Support:}


This research received no specific grant from any funding agency in the public, commercial, or not-forprofit sectors.

\section{Conflict of Interest:}

The Author declares that there is no conflict of interest.

\section{Availability of data and materials}

The dataset(s) supporting the conclusions of this article is(are) included within the article.

\section{References}

1. www.cancer.net/cancer-types/breast-cancer/statistics

2. Siegel R, Ma JM, Zou ZH, et al. Cancer statistics. CA Cancer J Clin.2014; 64:9-29

3. Houssami N, Abraham LA, Miglioretti DL et al. Accuracy and outcomes of screening mammography in women with a personal history of early-stage breast cancer. JAMA.2011;305(8):790-9

4. Patani N, Martin LA, Dowsett M. Biomarkers for the clinical management of breast cancer: international perspective. Int J Cancer.2013;133(1):1-13

5. Schwarzenbach H, Hoon DSB, Pantel K. Cell-free nucleic acids as biomarkers in cancer patients. Nat Rev Cancer.2011;11(426-437(2011)

6. Mandel P, Metais P. Blood plasma nucleic acids in humans. C R Acad Sci Paris.1948;142:241-

7. Leon SA, Shapiro B, Sklaroff DM, Yaros M et al.Free DNA in the serum of cancer patients and the effect of therapy. JCancer Res. 1977;37(3):646-50

8. Gormally E, Caboux E, Vineis $P$ et al.Review Circulating free DNA in plasma or serum as biomarker of carcinogenesis: practical aspects and biological significance. Mutat Res.2007;635(2-3):105-17

9. Jahr S., Hentze H., Englisch S. et al. DNA fragments in the blood plasma of cancer patients: quantitations and evidence for their origin from apoptotic and necrotic cells. Cancer Res. 200;161(4):1659-1665

10. Hiller, J., Brodner, G. \& Gottschalk, A et al. Understanding clinical strategies that may impact tumour growth and metastatic spread at the time of cancer surgery. Best Pract. Res. Clin. Anaesthesiol. 2013;27, 427-439

11. . Celec P, Vlková B, Lauková L et al. Cell-free DNA: the role in pathophysiology and as a biomarker in kidney diseases. Expert Rev Mol Med. 2018; 20:1-14.

12. Stötzer OJ, Lehner J, Fersching-Gierlich D et al.Diagnostic relevance of plasma DNA and DNA integrity for breast cancer.Tumour Biol. 2014;35(2):1183-91.

13. Spindler KL, Pallisgaard N, Andersen RF, et al. Circulating free DNA as biomarker and source for mutation detection in metastatic colorectal cancer. PLoS One. 2015;10(4): e0108247 
14. Gautschi O., Bigosch C., HuegliB., et al. Circulating deoxyribonucleic Acid as prognostic marker in non-small-cell lung cancer patients undergoing chemotherapy. J Clin Oncol. 2004;22 (20):41574164

15. Neeman E, Zmora O, Ben-Eliyahu S et al. A new approach to reducing postsurgical cancer recurrence: perioperative targeting of catecholamines and prostaglandins. Clin Cancer Res. 2012;18(18):4895902

16. Roxburgh, C. S., Horgan, P. G. \& McMillan, D. C. The perioperative immune/inflammatory insult in cancer surgery: Time for intervention? Oncoimmunology.2013; 2:e27324

17. Ni Choileain, N. \& Redmond, H. P. Cell response to surgery. Arch. Surg. 2006;141, 1132-1140

18. Saphner T, Tormey DC, Gray R. Annual hazard rates of recurrence for breast cancer after primary therapy. J ClinOncol.1996;14:2738-2746. (1996)

19. Marco Colleoni, Zhuoxin Sun, Karen N. Price, et al. Annual Hazard Rates of Recurrence for Breast Cancer During 24 Years of Follow-Up: Results from the International Breast Cancer Study Group Trials I to V.J ClinOncol. 2016;34(9): 927-935

20. Yu D, Tong Y, Guo X, et al. Diagnostic Value of Concentration of Circulating Cell-Free DNA in Breast Cancer: A Meta-Analysis. Front Oncol.2019;9:95

21. Crowley E., Di Nicolantonio F., Loupakis F. Liquid biopsy: monitoring cancer-genetics in the blood. Nat. Rev. Clin. Oncol.2013;10(8):472-484

22. Spellman P. T, Gray J. W, et al. 2014. Detecting cancer by monitoring circulating tumor DNA. Nat. Med.2014;20:474-475

23. Ravit Agassi, David Czeiger, Gad Shaked, et al. Measurement of Circulating Cell-Free DNA Levels by a Simple Fluorescent Test in Patients with Breast Cancer. American Journal of Clinical Pathology.2015;143:18-24

24. Li-Han Lin, Kuo-Wei Chang, Shou-Yen Kao et al. Increased Plasma Circulating Cell-Free DNA Could Be a Potential Marker for Oral Cancer. Int. J. Mol. Sci. 2018;19(11), 3303

25. Patrick J. Bastian, Ganesh S. Palapattu, SrinivasanYegnasubramanian et al. Prognostic Value of Preoperative Serum Cell-Free Circulating DNA in Men with Prostate Cancer Undergoing Radical Prostatectomy. Clin Cancer Res. 2007;13(18) >P:

26. He Y, Ma X, Chen K, et al. Perioperative Circulating Tumor DNA in Colorectal Liver Metastases: Concordance with Metastatic Tissue and Predictive Value for Tumor Burden and Prognosis. Cancer Manag Res.2020;12:1621-1630

27. Boysen, A. K., Wettergren, Y., Sorensen, B. S. et al. Cell-free DNA levels and correlation to stage and outcome following treatment of locally advanced rectal cancer. Tumor Biology.2017;1-6

28. Tan G, Chu C, Gui X, Li J, Chen Q. The prognostic value of circulating cell-free DNA in breast cancer: A meta-analysis. Medicine (Baltimore). 2018;97(13):e0197

29. Fleming CA, O'Leary DP, Wang J, Redmond HP et al. Association of Observed Perioperative Cell-Free DNA Dynamics With Early Recurrence in Patients with Colon Cancer. JAMA Surg.2019;4706. 
30. Hu W, Yang Y, Zhang L, et al. Post surgery circulating free tumor DNA is a predictive biomarker for relapse of lung cancer. Cancer Med. 2017;6(5):962-974

31. P. Jiang, Y. Lo, et al. The long and short of circulating cell-free DNA and the ins and outs of molecular diagnostics. Trends Genetics.2016; 32:6:360-371

32. Stroun M, Lyautey J, LederreyC, et al. About the possible origin and mechanism of circulating DNA apoptosis and active DNA release. ClinChimActa. 2001; 313:139-42

33. Uramoto, H., and Tanaka F. et al. Prediction of recurrence after complete resection in patients with NSCLC. Anticancer Res.2012; 32:3953-3960

34. Yang J, Cheng L, Zhang J, et al. Predictive value of circulating cell-free DNA in the survival of breast cancer patients: A systemic review and meta-analysis. Medicine (Baltimore). 2018;97(28):11417

35. Diehl F, Schmidt K, Choti MA, et al. Circulating mutant DNA to assess tumor dynamics. Nat. Med.2008;14(9):985-990

36. Cohen J.D, Li L., Wang Y.et al.Detection and localization of surgically resectable cancers with a multianalyte blood test. Science. 2018;359: 926-930

37. Cristiano S., Leal A., Phallen J.et al.Genome-wide cell-free DNA fragmentation in patients with cancer. Nature. 2019;570: 385-389

38. M. C. Liu1y, G. R. Oxnard, E. A. Klein , \& on behalf of the CCGA Consortium et al. Sensitive and specific multi-cancer detection and localization using methylation signatures in cell-free DNA. Annals of Oncology. 2020;31:6;745-759

\section{Tables}

\section{Table 1}

Clinico-pathological characteristics of patients in Disease free (DF) and Disease recurrence (DR) breast cancer 
Variables Total Disease free (DF)Breast

cancer within 5 years

No.

(percent) No. (percent)

63(100\%) 44(69.8\%)

No.
Disease Recurrence (DR) Breast cancer within 5 years

No. (percent)

19(30.1\%)

Gender

$\begin{array}{llll}\text { Female } & 61(96.8 \%) & 42(66 \%) & 19(3) \\ \text { Male } & 2(3.1 \%) & 2(3.17 \%) & 0\end{array}$

\begin{tabular}{llll}
$\begin{array}{l}\text { Age } \\
\text { (mean) }\end{array}$ & 56.4 & 56.4 & 56.5 \\
\hline$<50$ year & $30(47.6 \%)$ & $19(30.1 \%)$ & $10(15.8 \%)$ \\
\hline >50 year & $35(55.5 \%)$ & $25(39.6 \%)$ & $9(14.2 \%)$ \\
\hline T stage & & & \\
\hline TIS & $2(3.2 \%)$ & $2(3.2 \%)$ & 0 \\
\hline T1 & $19(30.1 \%)$ & $10(15.8 \%)$ & $9(14.2 \%)$ \\
\hline T2 & $35(55.5 \%)$ & $28(44.4 \%)$ & $6(9.5 \%)$ \\
\hline T3 & $7(11.1 \%)$ & $5(34.9 \%)$ & $2(3.2 \%)$ \\
\hline
\end{tabular}

Lymph

node

Positive $\quad 32(50.7 \%) \quad 22(35 \%)$

$10(15.8 \%)$

Negative $\quad 31(49.2 \%) \quad 22(35 \%)$

$9(14.2 \%)$

$p=1$

Subtypes

ER PR + $\quad 52(82.5 \%) \quad 40(63.4 \%)$

12(19.0\%)

$\mathrm{p}=0.012$

Her 2

$11(17.4 \%) \quad 5(7.9 \%)$

$6(9.5 \%)$

$p=0.076$

$\begin{array}{lll}\text { TNBC } & 6(9.5 \%) \quad 2(3.2 \%)\end{array}$

$4(6.3 \%)$

$\mathrm{P}=0.065$

\section{LVI} status

Positive 39(62\%) 30(47.6\%)

Negative $\quad 24(38.0 \%) \quad 14(22.2 \%)$

$9(14.2 \%)$

10(15.8\%)

$p=0.15$

Table 2 
Cox proportional hazard model to assess the impact of variables and cfDNA concentrations on recurrence free survival in breast cancer

Univariate Analysis

Variables

$\begin{array}{lll}P & \text { Hazard } & 95 \% \text { Confidence } \\ \text { value } & \text { ratio } & \text { Interval }\end{array}$

Lower

Upper
Multivariate analysis $\begin{array}{lll}P & \text { Hazard } & 95 \% \text { Confidence } \\ \text { value } & \text { ratio } & \begin{array}{l}\text { Interval } \\ \text { Into }\end{array}\end{array}$

Lower Upper

\begin{tabular}{|c|c|c|c|c|c|c|c|c|}
\hline $\begin{array}{l}\text { Age (<50 year vs >50 } \\
\text { year) }\end{array}$ & .326 & 1.571 & .638 & 3.868 & & & & \\
\hline $\begin{array}{l}\text { Tumour size (<2cm vs. } \\
>2 \mathrm{~cm})\end{array}$ & .009 & .294 & .118 & .733 & .006 & .216 & .072 & .644 \\
\hline $\begin{array}{l}\text { Grade (Grade1/2 vs. } \\
\text { Grade 3) }\end{array}$ & .289 & 1.747 & .622 & 4.901 & & & & \\
\hline T stage: & .059 & & & & & & & \\
\hline \multicolumn{9}{|l|}{ T0/1 } \\
\hline T2 & .2 & .300 & .111 & .813 & & & & \\
\hline T3 & .452 & .561 & .124 & 2.532 & & & & \\
\hline $\begin{array}{l}\text { Lymph node (positive } \\
\text { vs negative) }\end{array}$ & .791 & 1.129 & .459 & 2.780 & & & & \\
\hline $\begin{array}{l}\text { LVI (positive vs } \\
\text { negative) }\end{array}$ & .047 & .209 & .044 & .977 & .191 & .500 & .177 & 1.415 \\
\hline Subtype & .005 & & & & .011 & & & \\
\hline \multicolumn{9}{|l|}{ ERPR+ } \\
\hline HER2+ & .011 & 3.927 & 1.359 & 11.343 & .021 & 4.020 & 1.231 & 13.134 \\
\hline TNBC & .004 & 5.780 & 1.728 & 19.336 & .006 & 6.474 & 1.701 & 24.648 \\
\hline $\begin{array}{l}\text { Preop cfDNA } \\
(<480 \mathrm{ng} / \mathrm{ml} \text { vs. } \\
>480 \mathrm{ng} / \mathrm{ml})\end{array}$ & .000 & 6.888 & 2.468 & 19.218 & .624 & 1.555 & .266 & 9.103 \\
\hline $\begin{array}{l}\text { Postop cfDNA } \\
(<480 \mathrm{ng} / \mathrm{ml} \text { vs. } \\
>480 \mathrm{ng} / \mathrm{ml})\end{array}$ & .000 & 9.005 & 2.971 & 27.292 & .017 & 11.698 & 1.548 & 88.373 \\
\hline
\end{tabular}


Figures

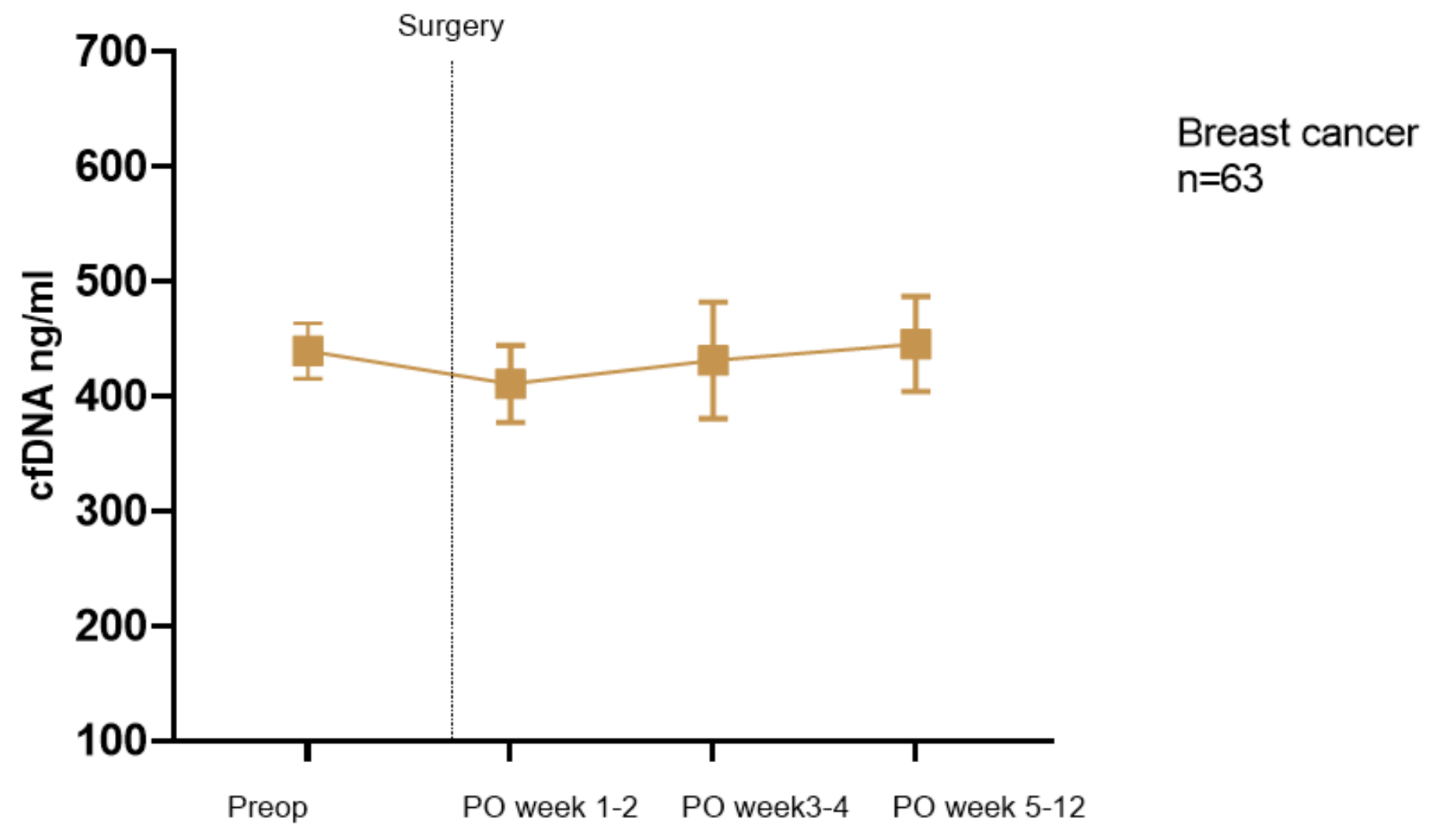

Figure 1

cfDNA concentration in peri-operative period in all breast cancer patients
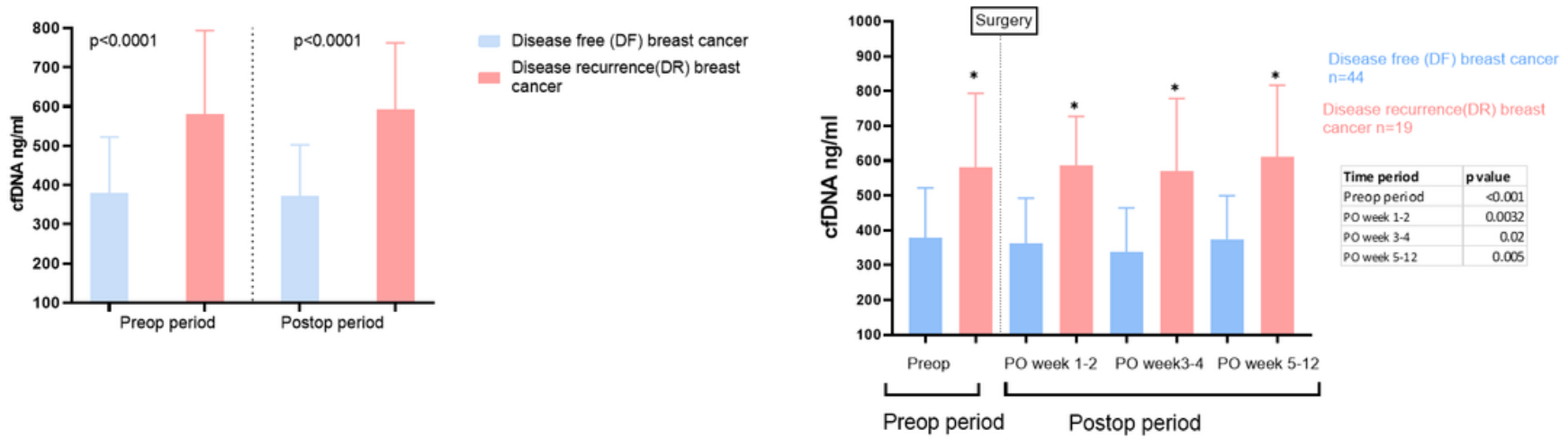

Figure 2

a. Cumulative cfDNA concentrations in subgroups (Disease free (DF) and Disease recurrence (DR) breast cancer) in perioperative time period $b$. Preoperative time period and serial postoperative cfDNA concentrations in subgroups (Disease free(DF) and Disease recurrence (DR) breast cancer) 


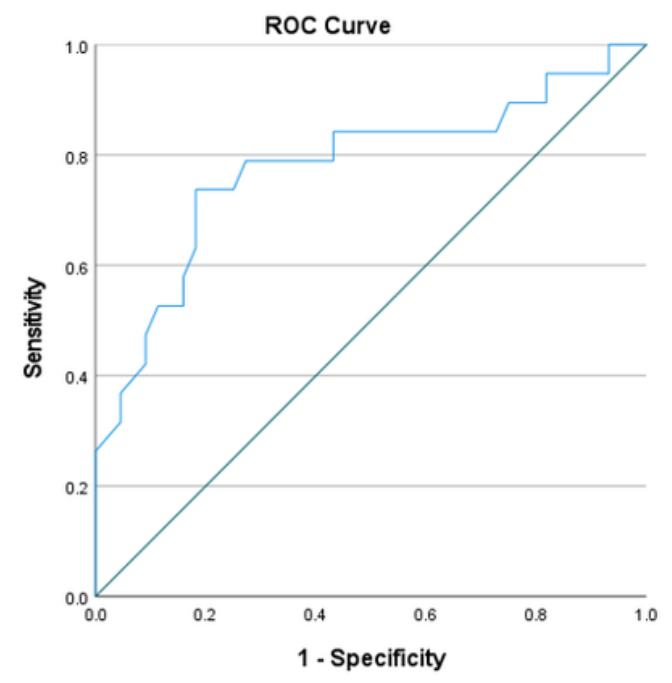

Diagonal segments are produced by ties.

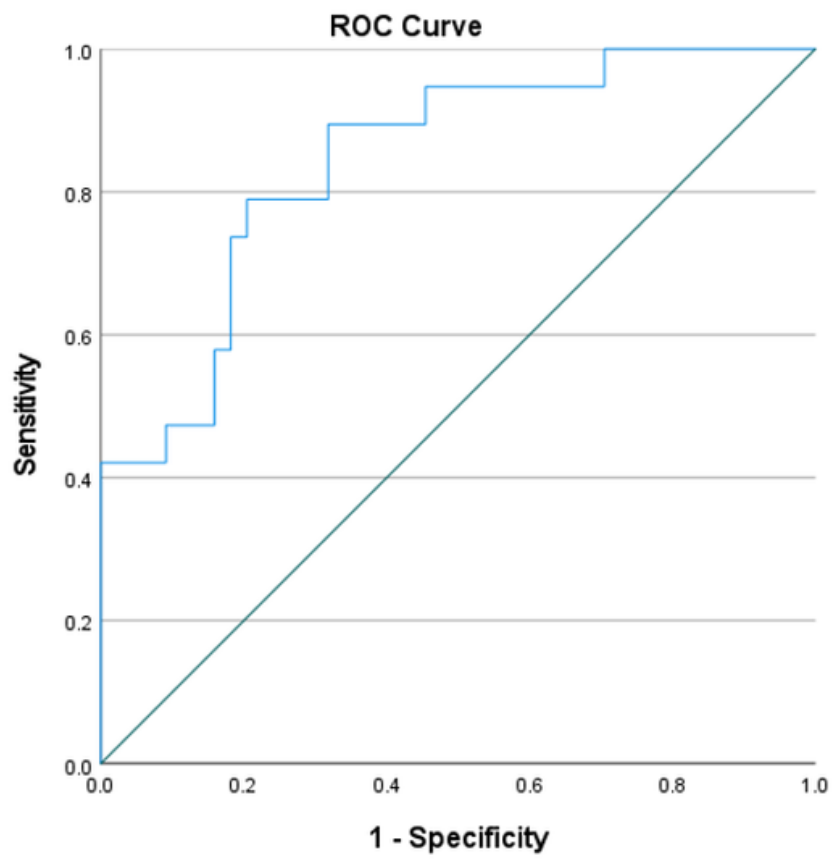

\section{Figure 3}

a. ROC curve analysis of pre-operative cfDNA concentrations in breast cancer. Area under curve (AUC) is 0.78 with $95 \%$ Confidence interval $0.64-0.91$. With cutoff value of $480 \mathrm{ng} / \mathrm{ml}$, sensitivity, and specificity of cfDNA concentrations in preop period was $74 \%$ and $82 \%$ respectively. b. ROC curve analysis of postoperative cfDNA concentrations in breast cancer. Area under curve (AUC) is 0.84 with $95 \%$ Confidence interval $0.74-0.95$. With cutoff value of $480 \mathrm{ng} / \mathrm{ul}$, sensitivity and specificity of cfDNA levels in postop period was $79 \%$ and $80 \%$ respectively.
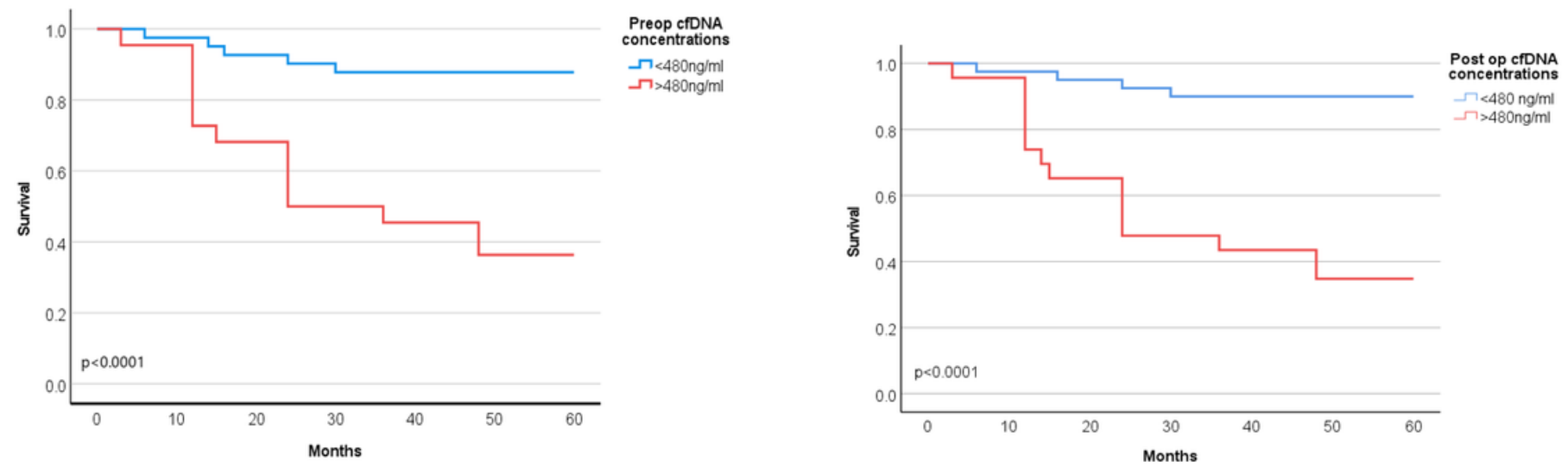

Figure 4 
a. Recurrence free survival analysis with Pre-operative cfDNA concentrations in breast cancer b. Recurrence free survival analysis with Post-operative cfDNA concentrations in breast cancer 